\title{
2-D Radiative Transfer Simulations with Angle-Dependent Partial Frequency Redistribution
}

\author{
A. B. Gorshkov \\ Sternberg Astronomical Institute, 119899 Moscow, Russia \\ P. Heinzel \\ Astronomical Institute, 25165 Ondřejov, Czech Republic
}

\begin{abstract}
We demonstrate how the angle-dependent redistribution function can be incorporated into the 2-D transfer modelling of solar prominences. Some preliminary numerical simulations have been performed and we present their results by comparing the emergent hydrogen $L \alpha$ line profiles computed with the angle-averaged and angle-dependent redistributions.
\end{abstract}

\section{Introduction}

Standard angle-averaged redistributions are currently used for Lyman lines in prominences and prominence-like structures, both in 1-D (Gouttebroze et al. 1993, Heinzel 1995), as well as in 2-D cases (Paletou 1995). Since 2-D transfer computations can explicitly account for strong anisotropies of the incident solar radiation, the angle-dependent approach seems to be more appropriate in such cases. In the present contribution we investigate this problem and demonstrate the differences between angle-averaged and more rigorous angle-dependent 2-D simulations for the case of prominences.

To achieve this, we have applied a 2-D code of Gorshkov (1996) to the problem of radiative transfer in solar prominences (Figure 1) for the case of a 4-level plus continuum HI atom. The main features of the code are: Multilevel Accelerated Lambda Iterations (MALI) scheme with Partial Frequency Redistribution (PRD) in resonance lines; modified long-characteristics method (timing is linearly proportional to the number of grid points) for 2-D solution of Radiative Transfer Equation (RTE); an ability to calculate angle- and height-dependent boundary conditions based on observational data.

\section{Basic Formulae}

\subsection{Angle-Dependent PRD}

In our calculations we used the redistribution function in the form:

$$
R\left(\nu^{\prime}, \nu, \Theta\right)=\gamma R_{I I}\left(\nu^{\prime}, \nu, \Theta\right)+(1-\gamma) \phi(\nu) \phi\left(\nu^{\prime}\right)
$$




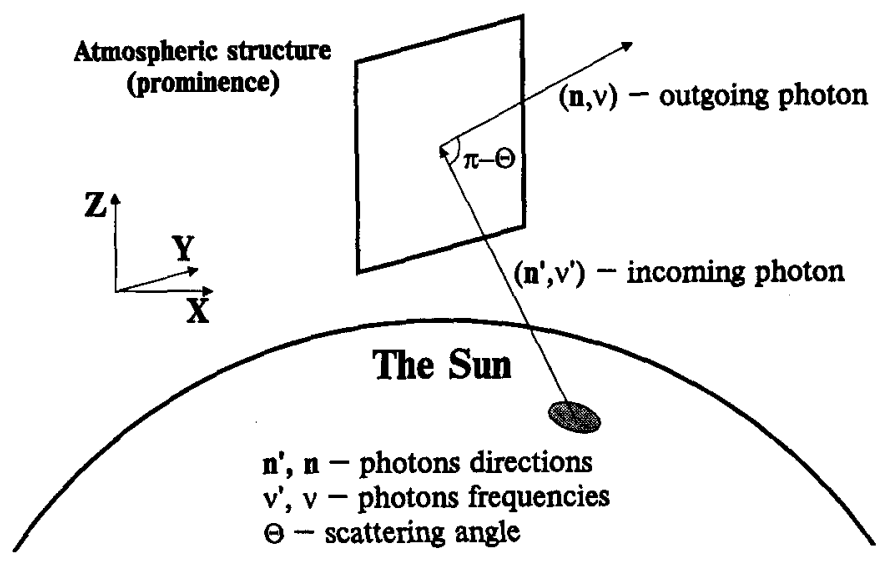

Figure 1. Photon scattering geometry.

where $\gamma$ is the coherence parameter, $\Theta$ is the scattering angle between directions of the incoming and the outgoing photons ( $\mathrm{n}$ ' and $\mathbf{n}$, correspondingly), and $R_{I I}$ represents Hummer's (1962) function for the case of purely coherent scattering in the atom's frame.

Similarly to the angle-averaged case, PRD effects are taken into account by introducing the ratio of emission and absorption coefficients for a given line transition $i j(i<j)$ :

$$
\rho_{\nu n} \equiv \frac{\psi_{\nu n}}{\phi_{\nu}}=1+\gamma \frac{n_{i}}{n_{j}} \frac{B_{i j}}{P_{j}}\left(\bar{R}_{I I}(\nu, n)-\bar{J}\right),
$$

where $n_{i}$ and $n_{j}$ are the populations of atomic levels $i$ and $j, B_{i j}$ is the Einstein coefficient for absorption, $P_{j}$ represents a probability for an atom to leave the level $j ; \bar{J}=(4 \pi)^{-1} \int_{0}^{\infty} \int_{0}^{4 \pi} I_{\nu^{\prime} n^{\prime}} \phi_{\nu^{\prime}} d \nu^{\prime} d \Omega^{\prime}$ is the mean integrated intensity and $\bar{R}_{I I}$ stands for the scattering integral (see Hubený 1985). Contrary to the standard PRD, $\rho$ now depends on the angle $\Theta$ because $\bar{R}_{I I}$ has the following form:

$$
\bar{R}_{I I}(\nu, n)=\left(4 \pi \phi_{\nu}\right)^{-1} \int_{0}^{\infty} \int_{0}^{4 \pi} R_{I I}\left(\nu^{\prime}, \nu, \Theta\right) I_{\nu^{\prime} n^{\prime}} d \nu^{\prime} d \Omega^{\prime},
$$

where the redistribution function is $\left(x, x^{\prime}\right.$ are frequencies counted from the line center and expressed in Doppler units):

$$
R_{I I}\left(x^{\prime}, x, \Theta\right)=\left\{\begin{array}{lc}
\frac{g(\Theta)}{\pi \sin \Theta} \exp \left\{-\left[\frac{1}{2}\left(x-x^{\prime}\right)\right]^{2} \csc ^{2} \frac{\Theta}{2}\right\} H\left[a \sec \frac{\Theta}{2}, \frac{1}{2}\left(x+x^{\prime}\right) \sec \frac{\Theta}{2}\right] \\
\frac{1}{\sqrt{\pi}} H\left(a, x^{\prime}\right) \delta\left(x-x^{\prime}\right) & \Theta<\pi \\
\frac{a}{2 \pi^{3 / 2}} \exp \left\{-\left(\frac{x-x^{\prime}}{2}\right)^{2}\right\}\left[\left(\frac{x+x^{\prime}}{2}\right)^{2}+a^{2}\right]^{-1} & \Theta=\pi
\end{array}\right.
$$


Here $H(a, x)$ is the Voigt function and $a$ the damping parameter. Examples of the function $R_{I I}\left(x^{\prime}, x, \Theta\right)$ are shown in Figure 2. Since the function varies very sharply with $\Theta$ and $x^{\prime}$, we used appropriate $\Theta$ - and $x^{\prime}$-dependent frequency quadratures for the evaluation of the scattering integral $\left({ }^{*}\right)$.
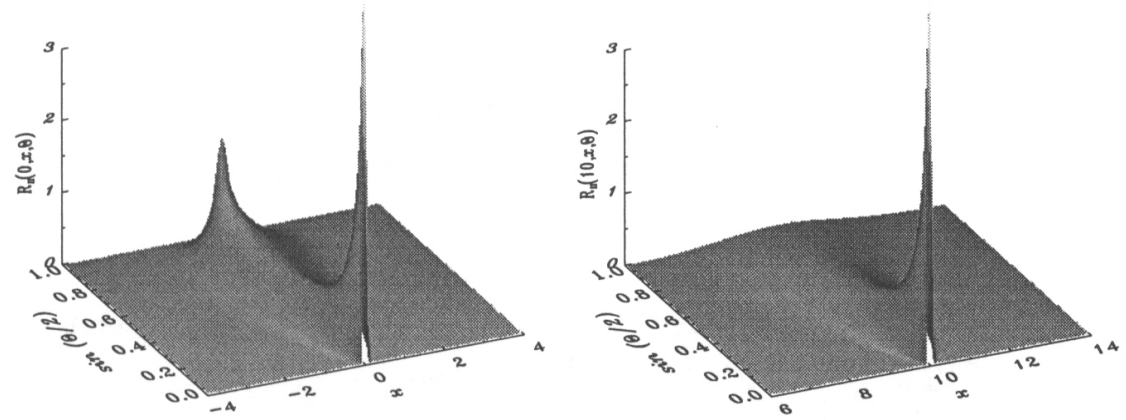

Figure 2. Function $R_{I I}\left(x^{\prime}, x, \Theta\right)$ for $x^{\prime}=0$ (left) and $x^{\prime}=10$ (right).

\subsection{Changes in the Formal Solution of RTE}

Since we need to know specific intensities $I_{\nu}$ to calculate the integral $\left({ }^{*}\right)$, the following changes in a standard Feautrier scheme of the formal solution of RTE are necessary:

$$
\begin{aligned}
& d^{2} u / d \tau^{2}=u-\bar{S}+d \Delta S / d \tau \\
& d^{2} v / d \tau^{2}=v-\Delta S+d \bar{S} / d \tau
\end{aligned}
$$

Here $u=\left(I_{\nu}^{+}+I_{-\nu}^{-}\right) / 2$ and $v=\left(I_{\nu}^{+}-I_{-\nu}^{-}\right) / 2$ are Feautrier variables; $\bar{S}=\left(S_{\nu}^{+}+\right.$ $\left.S_{-\nu}^{-}\right) / 2$ and $\Delta S=\left(S_{\nu}^{+}-S_{-\nu}^{-}\right) / 2$ represent averaged sum and difference of source functions in positive $(+)$ and negative $(-)$ directions. The scale of optical depths $\tau$ is calculated in a positive direction. To solve these transfer equations, we introduced corresponding changes in an improved Feautrier method of Rybicki and Hummer (1991).

\section{Results and Conclusion}

Using the above-described approach, we have computed 2-D transfer for the prominence model having the following parameters: dimensions $\Delta Z=2000$ $\mathrm{km}, \Delta Y=2000 \mathrm{~km}$, low boundary at the height $H=10,000 \mathrm{~km}$ above the solar surface, temperature $T=8000 \mathrm{~K}$, gas pressure $P_{\text {gas }}=0.05 \mathrm{dyn} / \mathrm{cm}^{2}$ turbulent velocity $V_{\text {turb }}=5 \mathrm{~km} / \mathrm{s}$. The angle-dependent incident radiation field was used similarly as in Gorshkov (1996), where other details of our numerical procedure are described. As a result of preliminary simulations, we present a comparison of emergent $L \alpha$ line profiles (taken in the center of the slab) for the cases of angle-averaged and angle-dependent PRD (see Figure 3). The main effect seen here is a lowering of the intensity in the line core. As a next step in 

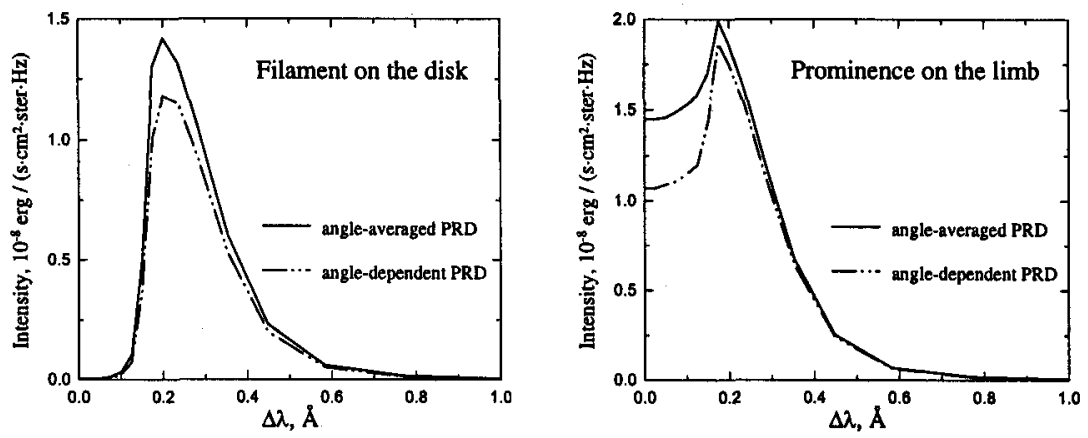

Figure 3. Calculated emergent profiles for the $\mathrm{L} \alpha$ line as seen on the disk (left) and on the limb (right).

this work we intend to demonstrate the influence of the angle-dependent PRD on spectral diagnostics of prominence plasmas.

Acknowledgments. A.B.G. greatly appreciates the support from the colloquium organizers which enabled him to attend the meeting. Travel expenses were covered from grant GACR 1199.

\section{References}

Gorshkov, A. B. 1996, in N. Mein and S. Sahal-Brechot (eds.), Science with THEMIS, Proc. Forum THEMIS, Observatoire de Paris-Meudon, p. 273

Gouttebroze, P., Heinzel, P. and Vial, J.-C. 1993, A\&AS, 99, 513

Heinzel, P. 1995, A\& A, 299, 563

Hubený, I. 1985, Bull. Astron. Inst. Czech, 36, 1

Hummer, D. G. 1962, MNRAS, 125, 21

Paletou, F. 1995, A\&A, 302, 587

Rybicki, G. B. and Hummer, D. G. 1991, A\&A, 245, 171 